\title{
Maladaptive Perfectionism in Asian International Students
}

\author{
Zainab Nazir ${ }^{1}$ and Vani Pillai ${ }^{1}$ \\ ${ }^{1}$ Service High School, Anchorage, AK, USA

\section{$\underline{\text { ABSTRACT }}$}

Because of the increased pressure on Asian international students to fit into American society while still maintaining ties to their country of origin, maladaptive perfectionism is very prominent in these students. This study's purpose was to investigate the impact of diversity on acculturation in addition to acculturation and parental influence on maladaptive perfectionism in Asian international students. A survey consisting of the Acculturative Stress Scale for International Students and The Frost Multidimensional Perfectionism Scale was sent out to all willing Asian international students. A preliminary analysis was conducted to analyze the impact of diversity on acculturative stress, and a multiple regression analysis was conducted to analyze the impact of parental influence and acculturation on maladaptive perfectionism. These analyses revealed that diversity has a positive effect on acculturative stress and when comparing parental influence and acculturation, parental influence has a greater impact on maladaptive perfectionism. Based on the current gap in research, these findings provide vital evidence regarding the positive impact of diversity in the process of acculturation.

\section{Introduction}

For many students, perfectionism, defined as the tendency to set excessively high standards and engage in overly critical self-evaluations, is seen as a highly positive trait that can help individuals achieve their goals (Kamakura, 1999). While in some cases this may be true, there is a multitude of factors that can make perfectionism extremely unhealthy and increase personal stress. One major factor that has been studied regarding this is the process of acculturation for international students. Acculturation, defined as the process of adapting to a new culture by making behavioral changes during the process, can have a negative impact on the students' well-being for a variety of reasons (Kamakura, 1999). These can include cultural stress, which can be a result of adjusting to a new culture, as well as prejudice from fellow students. These negative aspects of acculturation have been shown to result in a type of perfectionism referred to as maladaptive perfectionism, a concept that will be further explored later in this paper, but generally has detrimental effects on the welfare of students.

Johanna Nilsson and colleagues (2008) from the Division of Counseling and Educational Psychology, University of Missouri-Kansas City, cited the need for further investigation on the effects of maladaptive perfectionism in relation to acculturation in their article, "The Relationships Among Perfectionism, Acculturation, and Stress in Asian International Students." After conducting their study, in which the results showed perfectionism as a response to acculturation can result in high amounts of stress, they concluded that "International students in [different] geographical areas of the United States may have different experiences, especially in areas that are more culturally diverse... Future studies may provide additional support to our findings by confirming our results with other populations" (Nilson, Butler, Shouse \& Joshi, 2008). The preceding evidence constitutes the research gap that is to be addressed in this paper which is how the process of acculturation affects perfectionism in Asian international students specifically in the Anchorage School District (ASD) in Anchorage, Alaska. The results would be considerably distinct from an area in a different state because of Alaska's remoteness and smaller population. 
Research has shown that the city of Anchorage contains some of the most diverse schools in the United States. Chad Farrell (2018), Professor of Sociology at the University of Alaska Anchorage, states in the book Imagining Anchorage: The Making of America's Northernmost Metropolis, that the ASD accounts for twenty-seven of the thirty most diverse high schools in the nation, with even the schools not appearing in the top rankings having twice the diversity of the national average. As a result, a significant Asian population exists in the ASD. To be exact, Farrell notes that Asian students account for $10.8 \%$ of the overall school population which in comparison to the national average of $4.9 \%$ in public schools is notable (Farrell, 2018). He also states that Anchorage is regarded as a "mediumsized immigration post," with Asia accounting for five out of the ten largest origin groups. The study's findings suggest that there is an increased likelihood of Asian international students in the ASD. Because of the diverse environment in the Anchorage School District, this research will be beneficial as it will help identify whether a diverse environment can positively impact the process of acculturation resulting in less maladaptive perfectionism. In order to conduct an effective study that was feasible in the time given, but still gather a representative sample, the sample size was shortened to just one high school in the ASD.

\section{Literature Review}

Essential to this study are three interrelated factors: maladaptive perfectionism, parental influence, and the process of acculturation for Asian international students.

\section{Maladaptive Perfectionism}

Maladaptive perfectionism, characterized by a fear of failure and the hope of avoiding penalties and mistakes, is increasingly prevalent in Asian American students as seen in previous research (Yoon \& Lau, 2008 p. 92). As stated in "Maladaptive Perfectionism and Depressive Symptoms Among Asian American College Students: Contributions of Interdependence and Parental Relations," a peer-reviewed article written by accredited researchers Jaimin Yoon and Anna S. Lau (2008) at the University of California, Los Angeles, "Perception of high parental expectations and criticism show $[\mathrm{s}]$ strong associations with depression among Asian American college students" (p. 96). Their valuable research shows that maladaptive perfectionism in Asian students can be directly attributed to parenting styles and excessive pressure, making these two elements significant. This statement is further affirmed by Alison Ram (2005) at the University of Canterbury, who states, "Maladaptive perfectionism has a negative impact on academic achievement, while adaptive perfectionism [the ability to recover from failure] can have a positive impact" (p.14). These positive and negative impacts affect not only education but also mental health. The results of her study found that negative aspects of perfectionism were associated with higher levels of depression and anxiety (p. 85). Data provided by the preceding studies tend to support the importance of maladaptive perfectionism as a negative factor in the mental health of adolescents.

\section{Parental Influence}

Furthermore, another leading factor in the development of maladaptive perfectionism is parental influence. According to Holly Schiffrin and Miriam Liss (2017), researchers at the Department of Psychological Science, University of Mary Washington, maternal helicopter parenting, defined as the tendency for mothers to pay excessive attention to their child, can be linked to maladaptive perfectionism due to lack of confidence in skills for the child, which is a result of parental pressures. As asserted by D.E Hamachek in Psychology: A Journal of Human Behavior, unhealthy perfectionism can manifest in a family environment where love and praise are based on performance with parents withdrawing affection when performance is poor (as cited in Miller \& Neumeister, 2017). Specifically, parental influence is important in the context of Asian students because of the prevalence of authoritarian parenting, a type of parenting in which there are high expectations and little nurturance, in Asian cultures. According to a study done by Kathleen Kawamura (1999) at the University of Massachusetts, Amherst, in which she measured authoritarian parenting and its effect on perfectionism, Asian American students consistently rated their parents as more authoritarian 
than Caucasian students. The preceding data indicate that parental influence has a great impact on Asian students' perfectionism.

\section{Acculturation}

The final factor to be researched in this paper is acculturation, a leading factor for the perfectionism experienced by Asian international students (cited in Nilson et al, 2008). As stated by Johanna Nilson and colleagues (2008) in the Journal of College Counseling, the prevalence of academic achievement and college success in many Asian cultures can lead to increased perfectionism and stress in international students. The study's findings show that the increased perfectionism combined with prejudice from domestic students leads to high levels of stress in international students. However, these results are counter to research by Toshitaka Hamamura and Philip G. Laird (2014), from the Department of Psychology at Trinity Western University, which provides a new perspective on this argument. The results of their valuable study found that there was no difference in mental health among the domestic and international students, affirming that maladaptive perfectionism may not be culturally dependent. It is important to note that this experiment was done with an uneven ratio of domestic to international students in a small, private university which could account for the differing results.

Given the preceding context, it is apparent that the results of perfectionism as a response to acculturation can vary drastically from one location to another, making research in the Anchorage School District, specifically the sampled high school, vital to understanding and improving the lives of international students. This leads to the question to be addressed in the paper, "How do the highly diverse environment of Anchorage and parental influence impact the process of acculturation and maladaptive perfectionism in Asian international students?" The factors discussed above will be measured through a detailed survey and analyzed through multiple regression analysis.

\section{Methodology}

This study examined the relationship between diversity and acculturation and in turn, the impact of acculturation and parental influence on maladaptive perfectionism in Asian international students in the Anchorage School District. To optimize results and gather appropriate data, an ethnographic study was conducted with a quantitative and statistical analysis consisting of a multiple regression analysis, which will be discussed later in this paper. For conducting research on perfectionism in specific cultures, an ethnographic study was a viable option, as seen in multiple peerreviewed articles, one example being "Differences in Perfectionism Across Cultures: A study of Asian American and Caucasian Students" by Kathleen Kawamura. In this study, participants went through an intensive process for selection which included ethnicity and undergraduate status. For the purposes of the current paper, given the time frame and feasibility, the instrument being used, a voluntary survey, was sent out to students through educators, and students could make the decision to participate. Regardless of the nature of the survey being voluntary, it should be noted that the sample size was representative of the population, with 32 out of the 40 total Asian international students in the school responding.

\section{Participants}

Participants were informed of the purpose of the study in a participant consent form containing the necessary information about the objective of this research and a personal letter from the researcher. The form was submitted and approved by the Institutional Review Board to ensure it met the American Psychological Association's ethical guidelines and no harm would come to the participants.

Thirty-two international high school students from various countries in Asia participated in the current study. In terms of academic years, approximately 15\% were freshmen, 31\% were sophomores, 34\% were juniors, and 15\% were seniors. Regarding gender, 46\% $(n=15)$ of the sample was male and 53\% was female $(n=17)$. Participants represented 3 countries: Philippines, South Korea, Vietnam, with the highest population, 78\% $(n=25)$, being from the Philippines. 


\section{Instruments}

One key component that was researched in this paper was the process of acculturation for Asian international students. Previous literature has utilized multiple different scales to measure acculturation. Researchers at the Journal of College Counseling employed the American-International Relations Scale (AIRS- Sodowsky \& Plake, 1991) to measure acculturation in their peer-reviewed article (Nilsonn et al, 2008). This scale is a 34- item instrument consisting of 3 subscales which include Perceived Prejudice, Acculturation/Social customs, and Language Use. Higher scores on the AIRS indicate lower levels of acculturation which is different from most scales measuring acculturation where higher scores indicate higher levels. For the purposes of this research, this instrument was deemed too simplistic as it did not address all the different aspects of acculturation. In order to properly explore the impact of diversity on acculturation, a more comprehensive instrument was needed. To accomplish this, the Acculturative Stress Scale for International Students (ASSIS; Sandhu \& Asrabadi, 1994) was used. The ASSIS measured acculturative stress by using a 5- point Likert Scale ranging from a score of 1 (strongly disagree) to 5 (strongly agree) (Hamamura \& Laird, 2014). The seven subscales in ASSIS measured Perceived Discrimination, Homesickness, Perceived Hate, Fear, Culture Shock/Stress Because of Change, Guilt, and Nonspecific Concerns. This scale was chosen because it clearly aligned with the focus of this specific research. It addressed the key components of acculturation, especially the negative aspects, such as discrimination and hate, that can result in maladaptive perfectionism.

The second scale used in this study for evaluating the levels of perfectionism, more specifically maladaptive perfectionism, and parental influence, was The Frost Multidimensional Perfectionism Scale (MPS; Frost et al, 1990). F-MPS is a multi-faceted measure of perfectionism that utilizes six scales which include Concern over Mistakes, Personal Standards, Parental Expectations, Parental Criticism, Doubts about Actions, and Organization (Yoon \& Lau, 2008). These items are also rated on a 5-point Likert Scale similar to ASSIS. One limitation of F-MPS in relation to this study was that it did not specifically measure maladaptive perfectionism. To minimize this limitation, previous researchers have taken specific subscales and organized the data collected from those to come to a conclusion about maladaptive perfectionism. Professors Jaimin Yoon and Anna S. Lou (2008) calculated a maladaptive perfectionism composite by adding the mean of the responses on the items from the Concern Over Mistakes subscale and the Doubts about Actions Subscales. A similar approach was used in this study. Finally, to establish a parental influence composite, the Parental Expectations and Parental Concerns subscales from the F-MPS were used. This was a viable option for the purpose of this study because these subscales directly looked at parental influence in relation to perfectionism. The primary statistical tool utilized for the data analysis was a multiple regression analysis as it has been used consistently within this discipline for research on a multitude of variables (Nilson et al, 2008; Ra \& Trusty, 2016)

\section{Procedure}

Participants were informed of this study through educators, where the educators gave the students the option to take this survey. The survey was sent out through the English-language learners (ELL) counselors, who mentor international students from a wide range of areas in high schools across the district, but for this study, one high school. For the interest and credibility purposes of the participants, this survey was a voluntary survey and was sent out on Google Forms. It consisted of the Acculturative Stress Scale for International Students (ASSIS; Sandhu \& Asrabadi, 1994) and The Frost Multidimensional Perfectionism Scale (F-MPS; Frost et al, 1990). For replicability and generalizability purposes, the survey was sent out to the Asian international students in a high school in the ASD.

\section{Results}

\section{Preliminary Analysis}

In order to optimize results and analyze the data to its full extent, a preliminary analysis was conducted. This is displayed in Table 1, which presents the descriptive statistics including means and standard deviations for all the study variables. Higher scores indicated higher levels of each variable. The range of responses refers to the minimum and 
maximum scores a student could attain for each subscale. This was a critical part of the preliminary analysis as certain subscales consisted of more questions than others.

TABLE 1- MEANS AND STANDARD DEVIATIONS

\begin{tabular}{|c|c|c|c|}
\hline Variable & Sum Range & Mean & Standard Deviation \\
\hline F-MPS- PE & $5-25$ & 17.28125 & 5.35955207 \\
\hline F-MPS- PC & $4-20$ & 11.28125 & 4.587742259 \\
\hline F-MPS- MD & $13-65$ & 36.09375 & 12.56816094 \\
\hline ASSIS- PD & $8-40$ & 15.5625 & 5.333627344 \\
\hline ASSIS- H & $4-20$ & 12.65625 & 3.447611719 \\
\hline ASSIS- PH & $5-25$ & 9.71875 & 2.966887149 \\
\hline ASSIS- F & $4-20$ & 7.8125 & 2.668325257 \\
\hline ASSIS- S & $3-15$ & 7.09375 & 2.171804289 \\
\hline ASSIS- G & $2-10$ & 5.15625 & \\
\hline ASSIS- M & & & \\
\hline
\end{tabular}

Note- Higher scores indicate higher levels of each variable. F-MPS= Frost Multidimensional Perfectionism Scale; PE- Parental Expectations; PC = Parental Criticism; MD= Maladaptive Perfectionism; ASSIS- Acculturative Stress Scale for International Students; $\mathrm{PD}=$ Perceived Discrimination; H= Homesickness; $\mathrm{PH}=$ Perceived Hate; $\mathrm{F}=\mathrm{Fear}$; $\mathrm{S}=$ Stress Due to Cultural Shock/ Change; $\mathrm{G}=$ Guilt; $\mathrm{M}=$ Miscellaneous

\section{Primary Analyses}

After conducting the preliminary analysis, the second part of the data analysis, presented in Figure 1, consisted of finding a standardized way to examine the 7 different acculturation variables: Perceived Discrimination, Homesickness, Perceived Hate, Fear, Stress Due to Cultural Shock/Change, and Miscellaneous.

To achieve this standardized score, the response severity of each participant was calculated by dividing the mean response by the maximum score, seen in Figure 1. A basic assumption throughout this analysis was that rather than starting at 0 , all values of the response severity began at 2 because a 5-Point Likert Scale was utilized (the lowest number a participant could answer was "1" or "strongly disagree"). A primary purpose of the standardized scores was to determine whether or not diversity had an impact on aspects of acculturation such as hate and discrimination and 
to narrow down to the three most prevalent variables in order to conduct an effective multiple regression analysis. Through this, it was determined that in this specific sample population, homesickness, guilt, and stress due to cultural shock and change were the three (out of seven) highest scoring aspects of acculturation. This indicates that Asian international students at this high school tended to experience these aspects of acculturation more than others.

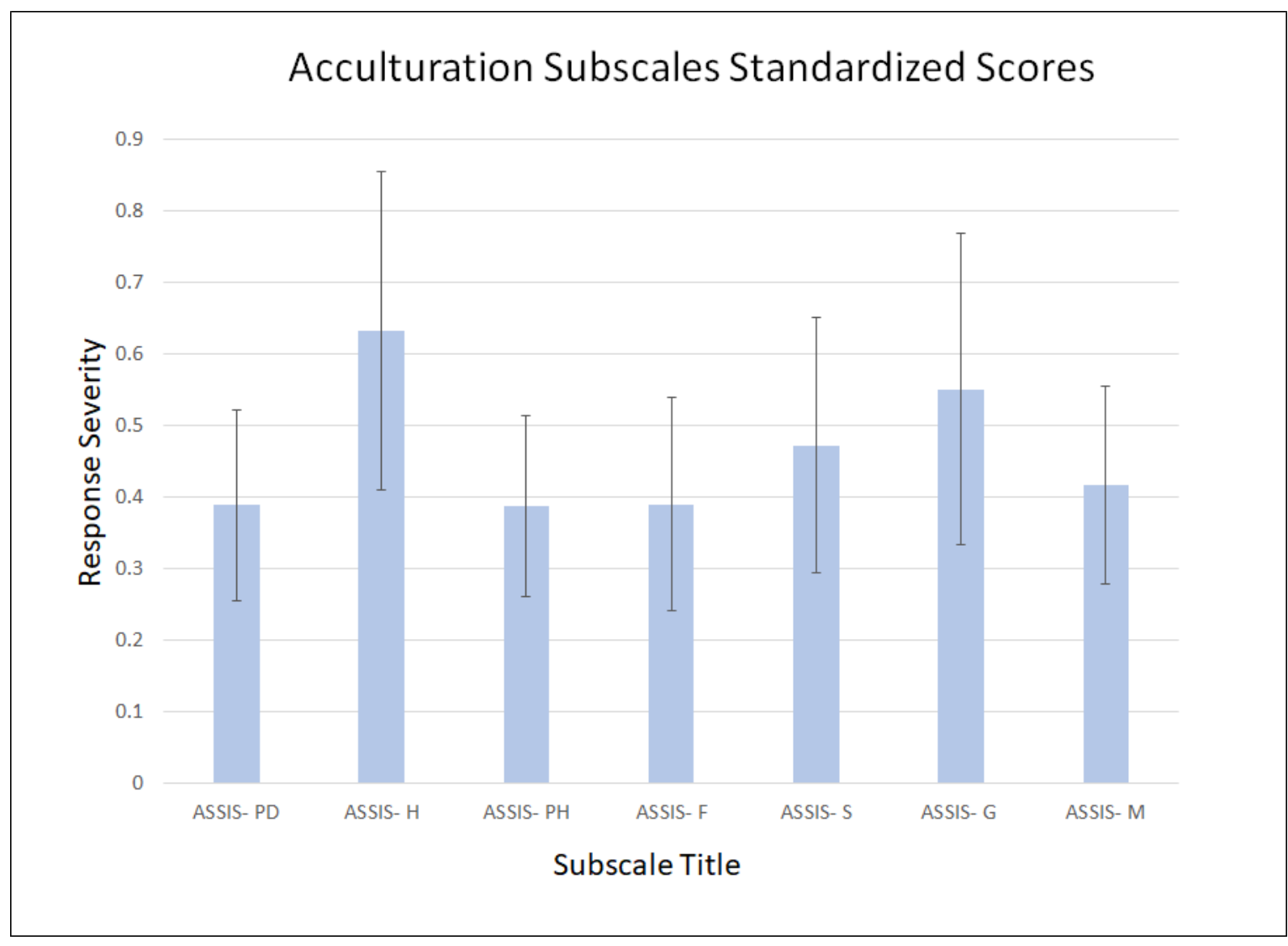

Figure 1

Note- All values start at .2 because of the 5-point Likert Scale used. Response severity was calculated by dividing the mean responses by the maximum score. ASSIS- Acculturative Stress Scale for International Students; PD= Perceived Discrimination; $\mathrm{H}=$ Homesickness; $\mathrm{PH}=$ Perceived Hate; F= Fear; $\mathrm{S}=$ Stress Due to Cultural Shock/ Change; $\mathrm{G}=$ Guilt; $\mathrm{M}=$ Miscellaneous

Ultimately, a multiple regression analysis was conducted to examine the impact of parental influence and acculturation on maladaptive perfectionism in Asian international students. The regression analysis was best suited for this study because it analyzed the independent variables of parental influence, cultural stress, homesickness, and guilt in relation to the dependent variable of maladaptive perfectionism. Stress, guilt, and homesickness were added in the first step, and parental influence was entered in the second step. The results of the regression analysis, seen in Table 2, showed that the acculturation variables predicted maladaptive perfectionism (Step 1). Parental influence explained the variance in maladaptive perfectionism beyond simply acculturation (Step 2). Whereas acculturation only accounted for $19 \%$ of the variance in maladaptive perfectionism, when combined with parental influence, they accounted for a $30 \%$ variance in maladaptive perfectionism. 
TABLE 2: RESULTS OF MULTIPLE REGRESSION ANALYSIS ON THE EFFECTS OF ACCULTURATION AND PARENTAL INFLUENCE ON MALADAPTIVE PERFECTIONISM

\begin{tabular}{|c|c|c|c|c|}
\hline Variable & $\mathbf{R}^{2}$ & SE & $\boldsymbol{\beta}$ & P-Value \\
\hline Step 1 & .19 & & & \\
\hline ASSIS-S & & 1.28 & .02 & .43 \\
\hline ASSIS-G & & 1.45 & .20 & .49 \\
\hline ASSIS-H & & .87 & .62 & .04 \\
\hline Step 2 & .30 & .26 & .56 & \\
\hline F-MPS- PI & & & & \\
\hline
\end{tabular}

Note- n=32, ASSIS- Acculturative Stress Scale for International Students; H=Homesickness; $S=$ Stress Due to Cultural Shock/ Change; G= Guilt; F-MPS= Frost Multidimensional Perfectionism Scale; PI- Parental Influence

It is important to note that the results of the regression analysis indicated that stress, guilt, and homesickness were not statistically significant ( $\mathrm{p}$-value $<0.05$ ). Conversely, the results of the subscale pertaining to parental influence were statistically significant, meaning that the regression analysis for this specific variable was of a greater significance than the acculturation variables. Further analysis regarding this finding will be explored in the discussion section.

\section{Discussion}

This study examined the impact of diversity on acculturation in addition to the influence of acculturation and parental influence on maladaptive perfectionism in Asian international students. The research gap addressed was the highly diverse environment of the Anchorage School District, specifically one high school. The study's findings indicate that a diverse environment results in an overall easier acculturation experience, as seen in Figure 1. Arguably the most difficult aspects of the transition to a new culture: perceived discrimination and perceived hate had relatively low scores compared to previous research, as seen in the study done by Nilson et al. Their results showed that there is a strong relationship between prejudice and stress for many international students (Nilson et al, 2008). Cultural stress was still an aspect of acculturation experienced by the sample population, but it was lower than the variables of homesickness and guilt, which scored the highest out of the seven acculturation variables studied. Given the previous context, it is clear that diversity may not have a major impact on more internalized aspects of acculturation that are not necessarily dependent on other individuals. An implication of the current study's findings is that further research is necessary for areas of homesickness and guilt for international students.

The second part of the research question addressed the aspect of maladaptive perfectionism as a result of parental influence and acculturation. Regarding these variables, the relationship among them is noteworthy, as seen in Table 2. The increase in variance for maladaptive perfectionism with the addition of parental influence is a notable result of the present research. This shows that even with lower acculturative stress, parental influence is a major contributor to maladaptive perfectionism. These findings are consistent with Yoon and Lau's study in which it was 
found that there was a "cultural basis underlying reports of higher levels of maladaptive perfectionism among Asian Americans college students relative to their non-Asian peers" (Yoon \& Lau 2008). Although their experiment focused on college students rather than high school students, the results were consistent with the present study. A possible explanation for these results is that maladaptive perfectionism in Asian international students can be strongly attributed to parental influence because of a high caliber of expectations in traditional Asian cultures (Nilson et al, 2008).

There are several limitations to this study that are necessary to address. Firstly, this research is only applicable to the sampled school's students and specifically only Asian international students. It is important to note that students in other high schools across the Anchorage School District may have different experiences and responses to the survey. Moreover, the current sample size was relatively small and only representative of predominantly East Asian countries, with the exception of Vietnam, which was regarded as a Southeast Asian country. Students from other Asian countries, for example, South Asian countries, may have different experiences. Furthermore, caution should be employed in grouping students with different backgrounds into one homogenous sample size. It is important to note that although in this study, all participants were regarded as one group, the results are not representative of all different Asian countries. Finally, regarding data results, it is critical to acknowledge that the acculturation variables were not seen as statistically significant when conducting the multiple regression analysis, meaning that the null hypothesis was not fully rejected. This could be due to several limitations such as response accuracy and limited sample size. However, the current results do provide support for the research hypothesis that maladaptive perfectionism is greatly influenced by acculturation even with the slight probability that the results occurred by chance. The present study indicates important implications for future studies in this field and the study's chosen high school in general.

To begin with, it is important to acknowledge that homesickness was highly prevalent among the sample studied, see Figure 1. This shows that there is a need for further research on this aspect of acculturation, especially in culturally diverse areas. Possible ways to address this issue of homesickness include social support and effective coping strategies. According to accredited researchers Young-An Ra and Jerry Trusty (2016) at Handong Global University and the Pennsylvania State University, acculturative stress, which can be due to homesickness, can be significantly reduced when Asian international students seek out social support. This is due to the fact that social support "facilitates the acculturation process and minimizes the acculturative stress of international students" (Ra \& Trusty, 2016). The previous literature combined with the results of the recent study suggests that social support is necessary to improve the acculturation process.

\section{Conclusions and Future Directions}

In terms of the Anchorage School District and the high school studied, more programs need to be put in place to connect international students to their country of birth while also making the transition to American culture easier. Most international students come to the United States knowing that acculturation is necessary but may not know the more negative aspects of it that they may face. A common example of this is becoming disconnected from their culture. If different cultures are normalized and taught in the classroom, surely this difficult process can become easier for students. Subsequently, the results of this study will be beneficial to education professionals who now have insight into the personal experiences of their students. Counselors, as well as teachers, will be better equipped to handle the encounters that many Asian international students face. The results of the current study indicate that acculturative experiences can vary depending on the area, making further research even more vital.

In conclusion, the study's findings suggest that a culturally diverse environment can positively impact the acculturation process for international students. However, further research in this field is necessary, especially in more remote areas such as Anchorage, Alaska. In order to provide quality education and support to international students, it is vital to understand their experiences, both positive and negative. Future studies may provide support to these findings by confirming the results with different areas, and more precisely, the individual experiences of Asian international students. 


\section{Acknowledgments}

I would like to thank my advisor Vani Pillai for helping me with this project.

\section{References}

Alaska Department of Education and Early Development. (2020). N/A high school. Retrieved from https://education.alaska.gov/compass/ParentPortal/SchoolProfile?SchoolID $=50660$

de Winter, J. F.C. and Dodou, D. (2010) "Five-Point Likert Items: t-test versus Mann-Whitney-Wilcoxon (Addendum added October 2012)," Practical Assessment, Research, and Evaluation: Vol. 15, Article 11.

Farrell, C. R. (2018). The Anchorage Mosaic: Racial and Ethnic Diversity in the Urban North. In Imagining Anchorage: The Making of America's Northernmost Metropolis (pp. 374-391).

Hamamura, T., \& Laird, P. G. (2014). The Effect of Perfectionism and Acculturative Stress on Levels of Depression Experienced by East Asian International Students. Journal of Multicultural Counseling \& Development, 42(4), 205-217. https://doi-org.sled.idm.oclc.org/10.1002/j.2161-1912.2014.00055.x

Hewitt, P.L., \& Flett, G.L. (1990). Perfectionism and Depression: a multidimensional analysis. Journal of Social Behavior and Personality, 5, 423-438

Kamakura, K. Y. (1999). Differences in perfectionism across cultures:: A study of Asian-American and caucasian college students (Master's thesis, U of Massachusetts Amherst). Retrieved from https://scholarworks.umass.edu/cgi/viewcontent.cgi?referer=https:/www.google.com/\&httpsredir=1\&article=3470\&context=theses

Mcleod, S. (2019). What a p-value tells you about statistical significance. Retrieved from https://www.simplypsychology.org/p-value.html

Miller, A. L., \& Neumeister, K. L. S. (2017). The Influence of Personality, Parenting Styles, and Perfectionism on Performance Goal Orientation in High Ability Students. Journal of Advanced Academics, 28(4), 313-344. https://doi-org.sled.idm.oclc.org/10.1177/1932202X17730567

Nilsson, J. E., Butler, J., Shouse, S., \& Joshi, C. (2008). The Relationships Among Perfectionism, Acculturation, and Stress in Asian International Students. Journal of College Counseling, 11(2), 147-158. https://doiorg.sled.idm.oclc.org/10.1002/j.2161-1882.2008.tb00031.x

Ra, Y., \& Trusty, J. (2017). Impact of Social Support and Coping on Acculturation and Acculturative Stress of East Asian International Students. Journal of Multicultural Counseling \& Development, 45(4), 276-291. https://doi-org.sled.idm.oclc.org/10.1002/jmcd.12078

Ram, A. (2005). The Relationship of Positive and Negative Perfectionism to Academic Achievement, Achievement Motivation, and Well-Being in Tertiary Students (Master's thesis, University of Canterbury). Retrieved from http://citeseerx.ist.psu.edu/viewdoc/download?doi=10.1.1.625.9900\&rep=rep1\&type=pdf 
Samuel, E. (2004). Racism in peer-group interactions: South Asian students' experiences in Canadian academe. Journal of College Student Development, 45, 407-42

Sandhu, D. S., \& Asrabadi, B. R. (1994). Development of an Acculturative Stress Scale for International Students: Preliminary Findings. Psychological Reports, 435-448.

Schiffrin, H., \& Liss, M. (2017). The Effects of Helicopter Parenting on Academic Motivation. Journal of Child \& Family Studies, 26(5), 1472-1480. https://doi-org.sled.idm.oclc.org/10.1007/s10826-017-0658-Z

Stober, J. (1998). The Frost Multidimensional Perfectionism Scale Revisited: More Perfect with Four (Instead of Six) Dimensions. Pergamon Journals, 24(2), 481-491. Retrieved from https://doc-0c-1g-apps-viewer.googleusercontent.com/viewer/secure/pdf/ugk5e0s8bc0tru7beo0s378m8a5ohe60/prd3c3bnfv49f48cbh48cf5krfijrn4q/1580407950000/gmail/ 13575710038684620105/ACFrOgB180yKMmY9EV4n9jnCQRon7XW X9yqZKwkBJ5DkNxMYV5NeyQH6wUyGeKX63CaFsmHcMDPdhdfzizZ2GLDQjHW5j0FqBKzhFVse K57F3PpT0GLFQsCqSNA=?print=true\&nonce=li56t9 miieat6\&user $=13575710038684620105 \&$ hash $=$ eg21d60gum69g $7 \mathrm{gn} 1 \mathrm{ht} 7 \mathrm{mtah} 1 \mathrm{fb} 3 \mathrm{fmm} 6$

Yoon, J., \& Lau, A. S. (2008). Maladaptive Perfectionism and Depressive Symptoms Among Asian American College Students: Contributions of Interdependence and Parental Relations. Cultural Diversity and Ethnic Minority Psychology, 14(2), 92-101. Retrieved from https://laulab.psych.ucla.edu/psych-laulab/wp-content/uploads/sites/2/2013/11/yoon-lau-2008.pdf 